\title{
Analysis And Optimization Of Car Interior Abnormal Noise
}

\author{
Zhang Wei $^{1, a}$ Liao Shuhuai ${ }^{1, b}$ Liu Li $^{2, ~ c ~}$ \\ 1. Guangxi University of Science and Technology, Guangxi 545006; \\ 2. China Automotive Technology and Research Center, Tianjin 300000 \\ aemail:814681098@163.com, bemail:liaohsh60@qq.com, ${ }^{c}$ email:348370299 @qq.com
}

\begin{abstract}
Keywords: Noise transfer function, dynamic stiffness, local dynamic stiffness
\end{abstract}
\begin{abstract}
This paper introduces the noise transfer function theory overview, After improvement proposed by the suspension of dynamic stiffness to reduce interior noise opinions, In an example of a compact car, We found the front peak problem by Accelerated conditions experimental test, Using the noise transfer function analysis and dynamic stiffness analysis, Combined with partial body modal, Find the cause of the problem in front peak, Proposed to strengthen the sub-frame dynamic stiffness to solve local problems in front peak, And to experimental verification, Indeed the final optimization, Better solve the problem of the front peak.
\end{abstract}

\section{Introduction}

Because of the global car ownership to swell, vehicle emission pollution and noise pollution to residents living environment cause the serious impact, vibration and noise of exhaust system directly affects the quality of harshness $(\mathrm{NVH})$ of vehicles, vehicle in the process of running, the more stable if the car more comfortable, and the smaller the in-car vibration noise, also means that the vehicle's harshness ( $\mathrm{NVH})$, the better the quality, and thus control and lower vibration noise is the major research and development institutions and automobile production enterprises attached great importance to the problem, the auto harshness (NVH) quality, is the important factors influencing the automobile market competition, directly affect the quality of the car, improve the performance of auto harshness (NVH) automobile production enterprises both at home and abroad is one of the important ways to improve its market core competitiveness ${ }^{[1]}$.

Working condition of this paper is aimed at accelerating test problems found in front of the peak, through the analysis on interior noise characteristics and order time, further through the noise transfer function analysis, dynamic stiffness analysis, modal analysis and the experimental method of combining the local deputy frame to determine after strengthening scheme to solve the problem in front of the peak, according to the experience of the project, considering the actual production costs and product production process technical issues (such as part moulds, assembly and processing technology, etc.), and through experimental tests, better solve the problem in front of the peak.

\section{Summary Of NTF}

Body Noise Transfer Function (Noise Transfer Function, NTF) ${ }^{[2]}$, mainly refers to the input load effect on car incentives and interior Noise is mainly a Function of output Noise. Pick up point Sound for the bodies of fluid-solid coupling system at a particular location for applying unit under a certain frequency simple harmonic motion, the interior noise reference points can be measured by the response of the body corresponding to the input load, known as the input to the point of reference for the sound pressure noise transfer function, research to identify the noise level of the vehicle noise transfer path, harshness $(\mathrm{NVH})$ characteristics, performance, the vibration noise of the vehicle design offers the comfort of a good performance. If role in the connection of the exciting force on the frequency response function for car body, installed in the interior sound pressure reference point position of the acceleration sensor and the microphone measuring the frequency response function is obtained, the transfer function, 


$$
H(\omega)=\frac{X(\omega)}{F(\omega)}
$$

In the structure of transmission noise transfer path related to the structure of three parts: engine mounting, the impedance of the body join points and body panels radiated noise characteristics. Structure of vehicle interior noise is caused by external incentive to panels vibration structure, at the same time, car panels and interior cavity radiation harm each other close to the car. When acoustic inherent in the frequency and excitation frequency, acoustic resonance will lead to aggravating the low-frequency noise "roar", comfort has a great influence on the passengers.

Free condition, regardless of the spoke to the effect of structural vibration, structural dynamic equation $^{[2]}$ :

$$
\left[M_{S}\right]\{\ddot{\mathrm{u}}\}+\left[K_{s}\right]\{u\}=\left\{F_{S}\right\}
$$

Type $\left[M_{S}\right]$ is the body structure type, mass matrix; $\left[K_{s}\right]$ for body structure stiffness matrix; $\{u\}$ for each node displacement vector; $\left\{F_{S}\right\}$ for exerting on the structure of the generalized force vector.

When sound solid coupling system dynamics equation, considering the fluid influence on structure, the air as the function of body panels are motivated by the external structure, body structure dynamics equation is expressed as:

$$
\left[M_{S}\right]\{\ddot{\mathrm{u}}\}+\left[K_{s}\right]\{u\}-[S]\left\{p^{a}\right\}=\left\{F_{S}\right\}
$$

Type $\left\{p^{a}\right\}$ for sound pressure on the boundary node vector.

Merge the above equation, get without considering damping sound solid coupling system dynamic equation:

$$
\left[\begin{array}{cc}
{\left[M_{S}\right]} & {[0]} \\
\rho[S]^{T} & {\left[M_{f}\right]}
\end{array}\right]\left\{\begin{array}{l}
\{\ddot{\mathrm{u}}\} \\
\ddot{\mathrm{p}}\}
\end{array}\right\}+\left[\begin{array}{cc}
{\left[K_{s}\right]} & {[S]} \\
{[0]} & {\left[K_{f}\right.}
\end{array}\right]\left\{\begin{array}{l}
\{u\} \\
\{p\}
\end{array}\right\}=\left\{\begin{array}{c}
\left\{f_{s}\right\} \\
\{0\}
\end{array}\right\}
$$

To solve the eigenvalue and eigenvector can get sound solid coupling modal frequency and vibration mode of the system.

\section{Problem Description}

A MPV vehicle on the road, while the vehicle noise testing in three full throttle (3G wot) conditions found in the front row have had obvious peak around $4300 \mathrm{r} / \mathrm{min}$, is also very obvious in the subjective feeling, in order to better solve the problem, improve the performance of the vehicle's harshness $(\mathrm{NVH})$ through various analysis methods are needed to find out the causes of the front peak, and find a method to solve the problem effectively. As shown in figure 1 to the driver on the right side pressure curve and the engine driver on the right side of the second order curve. The car engine for four-cylinder gasoline engine, and through the test results can be seen that the second order peak is due to the engine, the corresponding engine second order frequency is $143 \mathrm{~Hz}$, so after the analysis of the main consideration near $143 \mathrm{~Hz}$ frequency. 


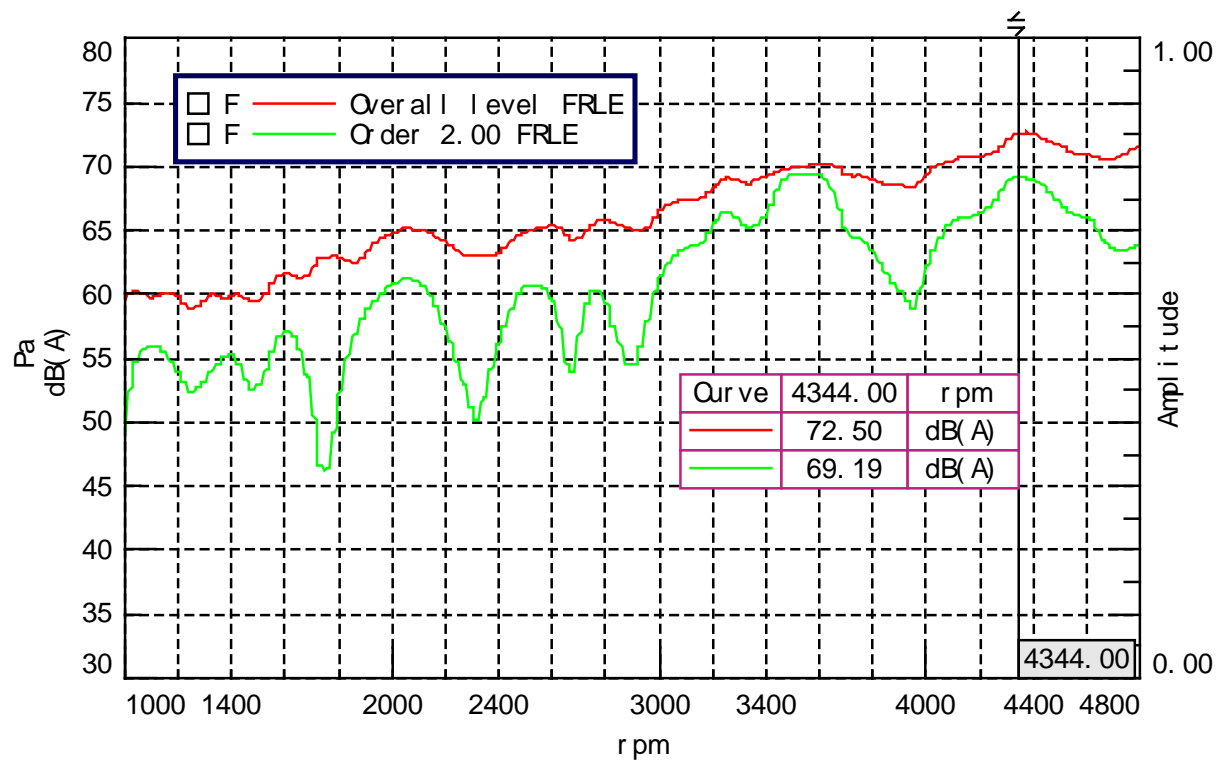

Figure 1 The driver on the right engine sound pressure curve and the driver on the right Quadradic

\section{Noise Transfer Function Analysis}

Join point noise transmission simulation analysis of the body, incentive point selection for body connection point, the response point selection in front of the driver's right ear, middle and rear passengers middle area to the right ear on the left side of the passenger, figure 2 for suspended three directions of the installation points after the noise transfer function curve.

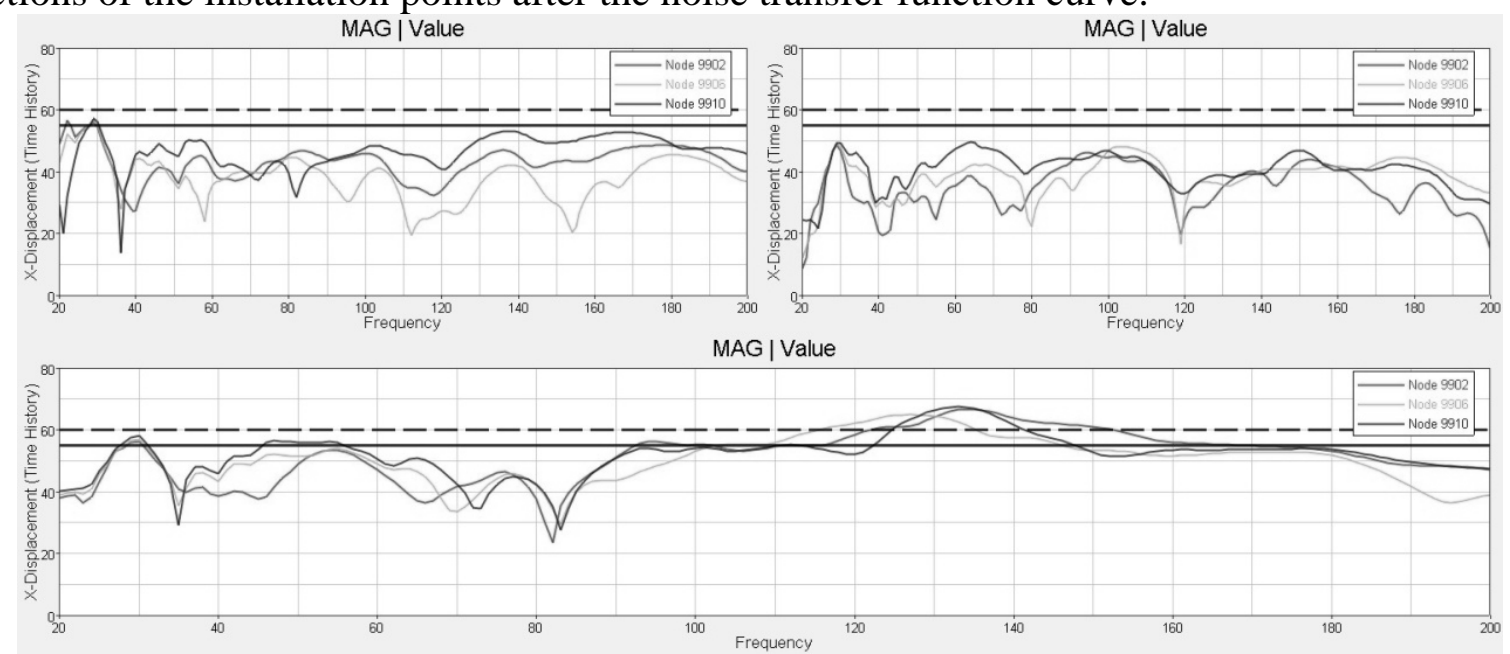

Figure 2 Noise in three directions after suspension mounting points of the transfer function curve

By above knowable, after mount $\mathrm{z}$ in $125-140 \mathrm{hz}$ to the front of the driver's right ear has obvious peak value, and is greater than the target value of $60 \mathrm{db}$.

\section{Dynamic Stiffness Analysis}

Comprehensive considering the calculation time and calculation accuracy, dynamic stiffness at the beginning of the simulation analysis for the car white body finite element model of connection points on the body dynamic stiffness analysis, in the mount installation point frequency sweep exciting force is applied to the unit, frequency sweep range of 20-200 hz. To calculate the dynamic stiffness curve as shown in figure 3. 


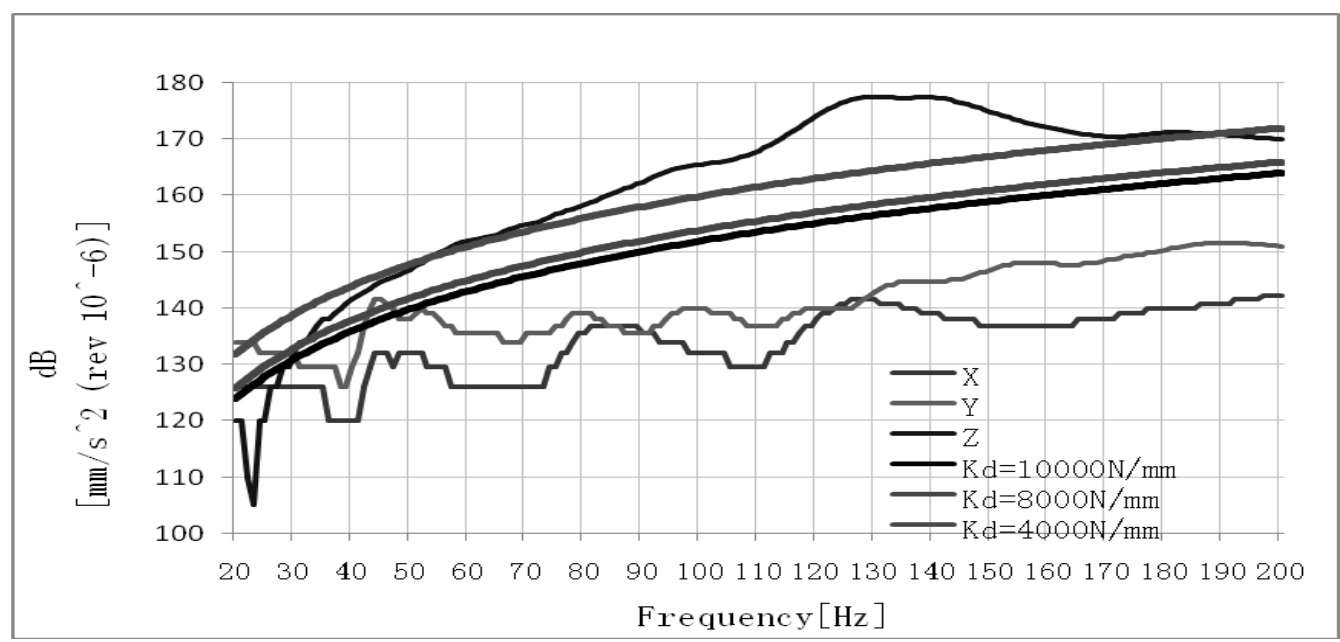

Figure 3 mount dynamic stiffness curve

After mount point $\mathrm{Z}$ to dynamic stiffness in the $80-160 \mathrm{hz}$ is far more than the target, but after the suspension of noise transfer function is also in the 125-125 hz didn't reach the goal request,

\section{The Optimization Scheme Is Put Forward}

Through the analysis of the noise of the suspension after following the transfer function and dynamic stiffness of suspension dynamic stiffness shortage is the main cause of the peak in the front row, the need to strengthen the local stiffness of the suspension after value to change the mode of torsion beam to avoid peak frequency, thus improving the front noise, according to the experience of the project, considering the actual production costs and product production process technical problems, finally determined the vice frame scheme, strengthening scheme as shown in figure 4, need through the analysis of the noise transfer function and dynamic stiffness to determine the optimal solution.

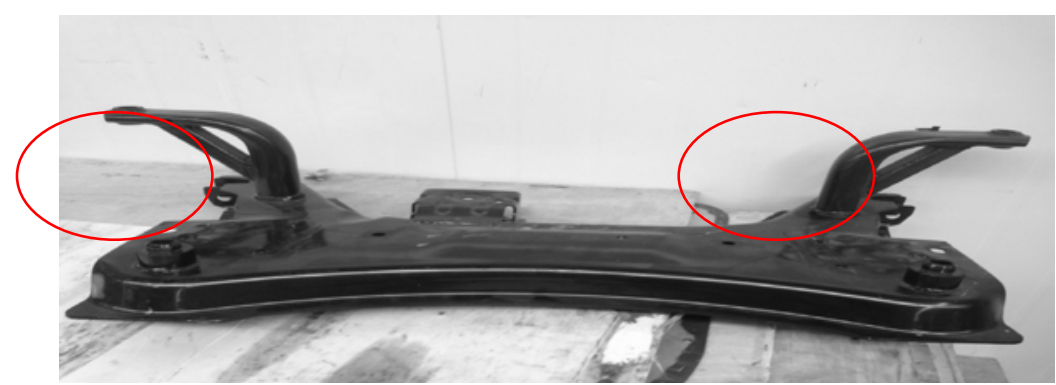

Figure 4 frame strengthening program

\section{Structure Optimization Analysis}

NTF scheme of optimization of strengthen pair of frame and suspension dynamic stiffness analysis, after analysis of the results as shown in figure 5, the solid line represents the optimized suspension before and after noise transfer function curve, dashed line represents the optimized suspension after noise transfer function curve. 


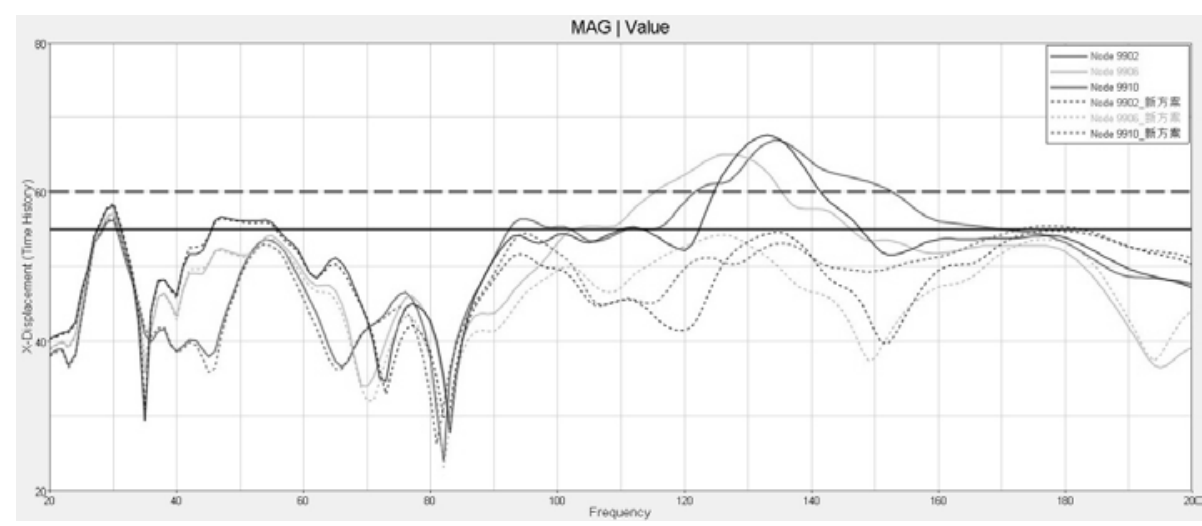

Figure 5 before optimization, after the suspension mounting points of the noise transfer function curve

\section{Optimization Experiment}

In combination with the practical situation of prototype, the optimization of the improved prototype vehicle interior noise test experiment, three block full throttle (3g wot) condition test, before and after improvement of auxiliary frame prototype in the actual drivers on the road of the right ear acoustic pressure curve test result is shown in figure 6, the fine line in order to optimize the former driver on the right side of sound pressure curve and the engine driver on the right side of the second order curve, bold lines for the optimized driver on the right side of sound pressure curve and the engine driver on the right side of the second order curve.

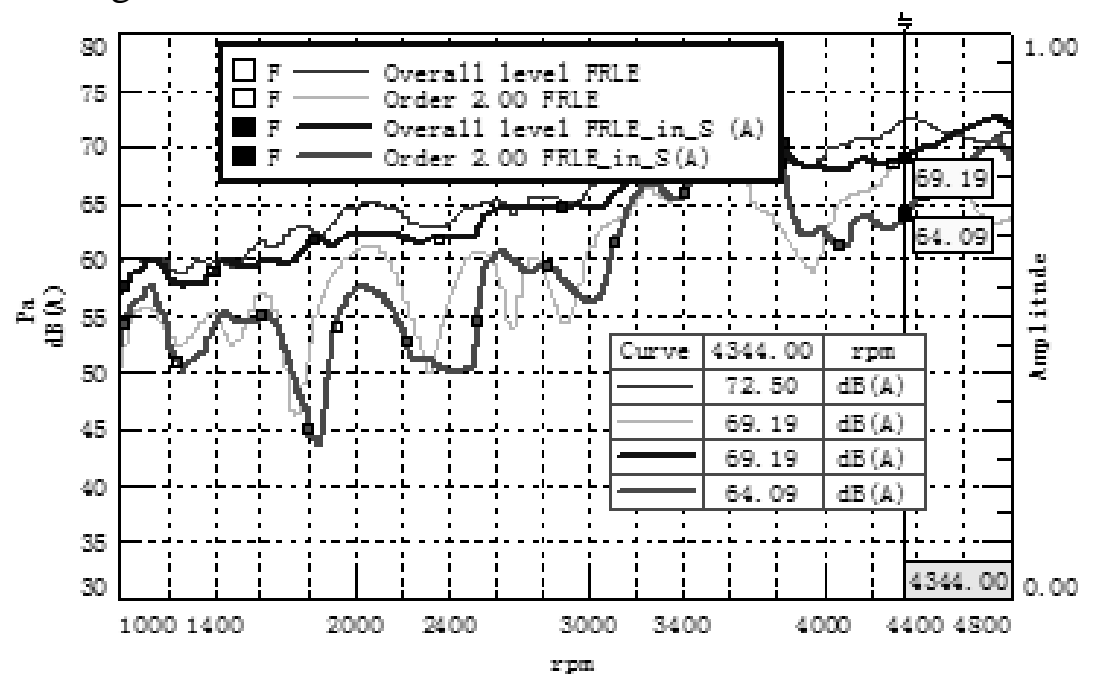

Figure6 Driver improvement program before and after the right side of the sound pressure curve and the engine driver's right side quadradic

By above knowable driver's right ear acoustic pressure peak value in $4344 \mathrm{r} / \mathrm{min}$ has been reduced, reduce by about $3 \mathrm{~dB}$, improved the problem in front of the peak. In the noise transfer function analysis combined with test methods, strengthen the local dynamic stiffness can effectively solve the peak in the front row.

\section{The Conclusion And Summary}

By CAE and testing method of combining the can in a relatively short period of time and under the control of economic cost, find out the peak problems, propose solutions to improve its harshness (NVH) performance.

By analyzing noise transfer function and dynamic stiffness, this paper analyzes the body suspension after noise transfer function of the installation points and the dynamic stiffness, and 
connecting with the local mode of car body, find out the corresponding frequency is obtained after the suspension mounting point dynamic stiffness is insufficient cause peak, considering the actual production costs and product production process technical problems, put forward to strengthen pair of frame scheme can effectively solve the problem in front of the peak.

\section{Reference}

[1]Pang Jian, Zhou Jianwen. Analysis of the structure of the noise in the car NTF problem rectification application [J]. Automotive technology.

[2] Kai Moer, Wolf Hyundai structure [M] Beijing: China Communications Press, 1987,360-375

[3] Nie Zuoxing, Body noise transfer global sensitivity analysis and robust optimal design function [D], Hunan University, Changsha, 201

[4] Xu Benwen, Jiao Qunying. Mechanical vibration and modal analysis of Beijing: Mechanical Industry Press, 1998: 50 to 51

[5] Wang Zhiliang, Liu Bo,Wang Lei. Car roar Mechanism Research and Analysis Method [J]. Noise and Vibration Control, 2008 (2): 79-81.

[6]Gong Chenbing, Wang Cunjie, Hu Li, Zhao Guang Fu. Based on MSC Nastran's front subframe stiffness [J] Computer Aided Engineering, 2013, S1:. 54-56.

[7]Song Haisheng, Shi Wenku, Guo Fuxiang, Shen Zhihong, Fang Deguang. Based subframe car floor vibration control analysis and optimization [J] Automotive Engineering, 2010,07: 601-604 + 610.

[8]Zhang Zhida, Xie Ran, Zhang WU. Optimization MTF FRF substructure synthesis method [J] Automotive Engineers, 2015,04: 38-41. 